\section{Research Square}

Preprints are preliminary reports that have not undergone peer review.

They should not be considered conclusive, used to inform clinical practice, or referenced by the media as validated information.

\title{
Genome-wide identification of DNA polymorphism in salt-tolerant and sensitive rice genotypes using double digest RADseq
}

\section{Rida Tariq}

National Institute for Biotechnology and Genetic Engineering

Muhammad Arif

National Institute for Biotechnology and Genetic Engineering

Hong-Bin Zhang

Texas A\&M University College Station: Texas A\&M University

JAUHAR ALI ( $\backsim$ J.Ali@irri.org )

International Rice Research Insititute https://orcid.org/0000-0002-3177-2607

\section{Research Article}

Keywords: Rice, Genotyping by sequencing (GBS), Single nucleotide polymorphisms (SNPs), Salt stress, Non-synonymous SNPs (nsSNPs)

Posted Date: February 8th, 2022

DOI: https://doi.org/10.21203/rs.3.rs-856946/v1

License: (9) (1) This work is licensed under a Creative Commons Attribution 4.0 International License. Read Full License 


\section{Abstract}

Salt-tolerant rice varietal development is necessary and the best approach to enhance crop productivity in areas with high salinity. To identify the genetic variation in stress-related genes in salt-tolerant genotypes, we carried out genotyping by sequencing (GBS) of seven rice lines with contrasting responses to salinity stress, including FL478 as a tolerant variety and IR64 as a sensitive check. The GBS reduces genomic complexity and enables us to obtain many SNP markers in the genome. A total of 7,980,092 high-quality single-end reads of 94 bp each were generated, and approximately $83 \%$ of the reads mapped to the rice reference genome (Nipponbare). We identified a total of 80,159 SNPs and 6,403 InDels among the seven rice genotypes. Forty-two percent of these SNPs were identified from the genic regions, and further, $27 \%$ were observed in the coding areas out of these genic SNPs. We used bioinformatics analysis to identify functional SNPs in the genes that changed the functions of proteins. We found variations between tolerant and sensitive genotypes by comparing the identified SNPs in coding regions. We found that 566 of the SNPs identified in the coding regions were non-synonymous (nsSNPs), affecting proteins. We identified 116 abiotic stress-related genes that contained 138 nsSNPs (between FL478 and IR64) in coding regions, thus directly affecting the proteins. Analysis indicated that these genes might contribute to the salt stress phenotype in rice varieties and the identified nsSNP markers that were useful to distinguish tolerant and sensitive genotypes. Our results revealed that the identified SNPs could be used as an essential source of genomic variability for improving and developing saline tolerant genotypes.

\section{Introduction}

Rice is an important cereal crop for human nutrition worldwide (Agarwal et al. 2016). It grows in areas where plenty of water is available with suitable temperatures (Batayeva et al. 2018). Over many decades, harsh environments had substantially reduced the yield of rice. It has been predicted that climate changes will increase the frequency of salinity, drought, high temperature, and flood (Mickelbart et al. 2015; Wang et al. 2016). Among these factors, salinity is known to inhibit plant growth, followed by excessive yield losses (Naveed et al. 2018). Soil salinity is one of the most serious threats to the productivity of this major agricultural crop, with adverse effects on all stages of plant growth (Al-Tamimi et al. 2016).

Rice often grows in coastal areas where various degrees of saline soils reduce its plant growth. Among cereals crops, rice is very sensitive to salinity. Most rice varieties can tolerate salinity up to $3 \mathrm{dS} / \mathrm{m}$. Many breeding programs worldwide use rice lines, such as Pokkali, Nona Bokra, etc., as salt-tolerant donors (Krishnamurthy et al. 2020). These lines have helped to improve the yield of elite genotypes under salt stress conditions (Negrão et al. 2013). Many studies have used molecular markers to identify QTLs related to salt tolerance by conventional mapping to distinguish sensitive and tolerant genotypes (Ali et al. 2014; Mondal and Ganie 2014). The use of conventional markers is a laborious and time-consuming process. Rice is the first cereal crop whose genome has been sequenced. The availability of the whole-genome sequence and improvement in next-generation sequencing (NGS) provides an opportunity to explore genetic differences in salt tolerance and sensitive varieties (Negrão et al. 2013). NGS technologies have helped identify SNP markers related to QTLs or target genes (Tiwari et al. 2016). Many rice projects aimed to provide vast amounts of information on the sources of genetic variation of diverse germplasm. The Rice SNP-Seek Database identified 20 million SNPs in the 3K rice genomes project by aligning with the rice reference Nipponbare genome. The outcome of this project will be helpful for the identification of novel allelic variants for crop improvement (Alexandrov et al. 2015).

NGS technologies could detect several variants in an efficient, rapid, and economical manner. These variants are significant reasons for phenotypic differences between different accessions (Ali et al. 2018). The SNPs and InDels identified have been widely used in fine mapping, QTL analysis, and markerassisted selection. Many SNPs has facilitated fine linkage analysis and genome-wide association study (Hayashi et al. 2006). The variants within the gene coding regions could affect the coding proteins, thus changing their function positively or negatively. Therefore, the presence of variants that lead to a change in gene function(s) is essential for studying the causes of phenotypic differences (Jain et al. 2014).

Several NGS platforms have been developed and widely used for SNP discovery in recent years (Sahu et al. 2020). One straightforward approach is to prepare GBS libraries with decreasing genomic complexity to attain many SNPs and a high read coverage by using restriction enzymes, followed by restricted DNA library construction. It allows the regions flanking only the restriction sites to be sequenced, thus dramatically reducing genomic complexity and sequencing cost. Furthermore, the sequenced genomic portion is highly stable within species because restriction sites are mostly conserved across genotypes (Zhao et al. 2019). Thus, it makes the GBS procedure most suited to analyzing large numbers of SNPs within a population for genetic mapping and genomics-assisted breeding. Furthermore, by selecting appropriate restriction enzymes that have fewer cutting sites in the repetitive regions, enriched SNP coverage can be obtained for the coding regions of the genome (Chung et al. 2017). In comparison with RADseq methods, double digest Restriction site-Associated DNA sequencing (ddRADseq) allows high efficiency, flexibility, and robustness with a low cost (Peterson et al. 2012). Genetic variations in abiotic stress-related genes are already reported; however, the breeding progress using these genes is plodding. Therefore, it is essential to directly sequence rice lines selected for particular breeding objectives to identify genes and QTLs for the targeted traits, for example, salt tolerance and their applications for enhanced and accelerated breeding (Zhao et al. 2011).

We have genotyped seven rice breeding lines showing differential responses to salt stress using ddRADseq technology. The objective was to identify the loci containing SNPs associated with salt tolerance, hence differentiating tolerant genotypes from sensitive ones for salt tolerance breeding.

\section{Materials And Methods}

Rice materials

Our previous study selected seven rice lines (Tariq et al. 2019) based on their responses to salinity at the seedling stage. Of these seven lines, four were salt tolerant and three salt sensitive (Table 1). 
Table 1

Salt stress responses of the rice lines used for this study

\begin{tabular}{|c|c|c|c|c|c|c|c|c|}
\hline \multirow[t]{2}{*}{ Original name } & \multirow[t]{2}{*}{ Parental cross } & \multirow[t]{2}{*}{ Designation } & \multirow{2}{*}{$\begin{array}{l}\text { Given } \\
\text { name }\end{array}$} & \multirow[t]{2}{*}{ Source } & \multicolumn{3}{|c|}{ SES scoring } & \multirow{2}{*}{$\begin{array}{l}\text { Salinity } \\
\text { tolerance }\end{array}$} \\
\hline & & & & & $\begin{array}{l}50 \\
\mathrm{mM}\end{array}$ & $\begin{array}{l}100 \\
\mathrm{mM}\end{array}$ & $\begin{array}{l}150 \\
\mathrm{mM}\end{array}$ & \\
\hline \multirow{2}{*}{$\begin{array}{l}\text { HHZ 8-SAL9- } \\
\text { DT1-Y1 }\end{array}$} & HHZ x Phalguna & \multirow{2}{*}{$\begin{array}{l}\text { GSR IR1-8-S9- } \\
\text { D1-Y1 }\end{array}$} & \multirow[t]{2}{*}{ SAL-9 } & \multirow[t]{2}{*}{ IRRI } & \multirow[t]{2}{*}{3} & \multirow[t]{2}{*}{5} & \multirow[t]{2}{*}{5} & \multirow{2}{*}{$\begin{array}{l}\text { Moderately } \\
\text { tolerant }\end{array}$} \\
\hline & (Ali et al, 2017) & & & & & & & \\
\hline \multirow{2}{*}{$\begin{array}{l}\text { HHZ 5-SAL10- } \\
\text { DT2-DT1 }\end{array}$} & $\mathrm{HHZ}$ x OM1723 & \multirow{2}{*}{$\begin{array}{l}\text { GSR IR1-5-S10- } \\
\text { D2-D1 }\end{array}$} & \multirow[t]{2}{*}{ SAL-10 } & \multirow[t]{2}{*}{ IRRI } & \multirow[t]{2}{*}{3} & \multirow[t]{2}{*}{3} & \multirow[t]{2}{*}{3} & \multirow[t]{2}{*}{ Tolerant } \\
\hline & (Ali et al, 2017) & & & & & & & \\
\hline \multirow{2}{*}{$\begin{array}{l}\text { HHZ 5-SAL10- } \\
\text { DT1-DT1 }\end{array}$} & $\mathrm{HHZ} \times \mathrm{OM} 1723$ & \multirow{2}{*}{$\begin{array}{l}\text { GSR IR1-5-S10- } \\
\text { D1-D1 }\end{array}$} & \multirow[t]{2}{*}{ SAL-11 } & \multirow[t]{2}{*}{ IRRI } & \multirow[t]{2}{*}{5} & \multirow[t]{2}{*}{7} & \multirow[t]{2}{*}{7} & \multirow[t]{2}{*}{ Sensitive } \\
\hline & (Ali et al, 2017) & & & & & & & \\
\hline \multirow{2}{*}{$\begin{array}{l}\mathrm{HHZ} 11-\mathrm{SAL6}- \\
\mathrm{Y} 1-\mathrm{Y} 1\end{array}$} & HHZ x IR64 & \multirow{2}{*}{$\begin{array}{l}\text { GSR IR1-11-S6- } \\
\text { Y1-Y1 }\end{array}$} & \multirow[t]{2}{*}{ SAL-12 } & \multirow[t]{2}{*}{ IRRI } & \multirow[t]{2}{*}{7} & \multirow[t]{2}{*}{7} & \multirow[t]{2}{*}{7} & Sensitive \\
\hline & (Ali et al, 2017) & & & & & & & \\
\hline FL478 & IR29 x Pokkali & FL478 & FL478 & IRRI & 3 & 3 & 3 & Tolerant \\
\hline & (Thomson st al, 2010) & & & & & & & \\
\hline Pokkali & Landrace & Pokkali & Pokkali & IRRI & 3 & 3 & 3 & Tolerant \\
\hline IR64 & $\begin{array}{l}\text { Came from } 19 \text { traditional rice varieties. (Mackill } \\
\text { and Khush 2018) }\end{array}$ & IR64 & IR64 & IRRI & 7 & 7 & 7 & Sensitive \\
\hline
\end{tabular}

Preparation of ddRADseq library and sequencing

Genomic DNA of the seven rice lines was extracted from 14-day-old seedlings using the CTAB (cetyl trimethyl ammonium bromide) method (Tariq et al. 2019). Genomic DNA was digested with two restriction enzymes, BamHI and $\mathrm{MluCl}$, for sequencing the BamHI site flanking sequences. The ddRADseq library was constructed for each line for single-ended sequencing using the Illumina HiSeq 2000 platform to generate 100-base-long reads. The FASTQ files of the reads were first trimmed to remove adapters and then the quality of the reads was checked using FastQC V0.63.

Sequence alignment and variant calling

The sequenced reads were filtered based on mapping quality $\geq 20$ but the low-quality reads were filtered out with mapping quality $\leq 20$ using NGS: QC Toolkit v2.3 (Patel and Jain 2012). The rice cv. Nipponbare reference genome IRGSP-1.0 pseudomolecules were downloaded from the Rice Genome Annotation Project (http://rice.plantbiology.msu.edu/) (Kawahara et al. 2013). The clean reads were aligned to the rice reference genome using BWA1.2.3 tool (Li and Durbin 2009) with default parameters. The Picard tool v1.126.0 (https://broadinstitute.github.io/picard/) was used to sort the aligned reads in the Sequence Alignment/Map (SAM) file by using SortSam function or plugin. The sorted SAM files were converted to Binary Alignment/Map (BAM) files using SAMtools v2.1 (Li et al. 2009). Variant calling was performed using Unified Genotyper of GATKTools v3.3 (DePristo et al. 2011) with a base quality > Q30. To minimize false-positive variants and retain high-quality variants, the variant calling file (VCF) was further filtered based on read depth $>10$ and allele frequency $>0.8$ using the Select Variants of GATKTools (DePristo et al. 2011). The quality of the identified SNPs and InDels was visualized using Integrative Genomics Viewer (IGV) (Robinson et al. 2011).

Variant annotation

The identified filtered variants in the rice genome were annotated using the rice7 database (https://sourceforge.net/projects/snpeff/files/databases/v4_2/) and SnpEff V4.2 tool (Cingolani et al. 2012). The locations of the SNP and InDel variants in the genic and intergenic regions were determined using the SNiPlay online tool (Dereeper et al. 2015). Based on the locations of the SNPs and InDels, the genic regions were further categorized into exons, introns, 3'UTRs, 5'UTRs, splice sites, and coding regions. The variants identified in the coding regions were further separated as synonymous (with no effect on amino acid), non-synonymous (with effects on amino acid), stop loss (the existing stop codon lost), stop gain (introduction of a new stop codon), start loss (the existing start codon lost), and start gain (introduction of a new start codon). The effect of a substituted amino acid on protein functions was determined by SIFT 4G (Vaser et al, 2016) using the rice IRGSP-1.0.23 genome build (http://sift.bii.a-star.edu.sg/index.html). This algorithm classified the presence of nonsynonymous SNPs as tolerated (having no effect on protein function) or deleterious (having the potential to change protein function).

Identification of non-synonymous SNPs (nsSNPs) in the salt stress genes

The nsSNPs were filtered from the annotated file, retaining only those nsSNPs that were polymorphic and deleterious by function between the tolerant (FL478) and sensitive genotype (IR64). Furthermore, only those nsSNPs were kept that were specifically located in the coding regions of loci associated with salt tolerance. The functions of the identified genes were obtained from Rice Genome Annotation Project (http://rice.plantbiology.msu.edu/), Phytozome the Plant Genomic Resource (http://phytozome.jgi.doe.gov/), and uniprot.org websites.

SNP validation 
The identified SNPs were validated by downloading the previously reported SNPs of Pokkali and IR64 (Jain et al. 2014) from the Rice SNP Discovery database (http://www.nipgr.res.in/mjain.html?page=ricesnp). The identified nsSNPs in coding regions were also validated with the available nsSNPs of FL478, Pokkali, and IR64 in the Rice SNP-Seek Database (Mansueto et al. 2017).

Genetic analysis

A distance-based neighbor-joining tree of high-quality nsSNPs was constructed using DARwin V6.0.1 (Perrier and Jacquemoud-Collet 2006). In addition, SNiPlay V4.2 generated genotyping matrices of high-quality SNPs.

\section{Results}

Responses of the seven rice lines to salt stress

The seven rice lines were further screened with three salt concentrations (i.e., 50, 100, and $150 \mathrm{mM}$ ) along with controls. All seven lines were evaluated as tolerant or sensitive based on the modified Standard Evaluation System (SES) score (Gregorio et al. 1997) by comparing the visual response of leaves of these genotypes with control checks under $150 \mathrm{mM} \mathrm{NaCl}$ (Tariq et al. 2019). Under this salt stress, SAL-9 (GSR IR1-8-S9-D1-Y1 = HHZ8-SAL9-DT1-Y1) and SAL-10 (GSR IR1-5-S10-D2-D1 = HHZ5-SAL10-DT2-DT1) were evaluated as tolerant because the leaves showed no sign of salt injury. In contrast, SAL-11 (GSR IR1-5S10-D1-D1 = HHZ5-SAL10-DT1-DT1) and SAL-12 (GSR IR1-11-S6-Y1-Y1 = HHZ11-SAL6-Y1-Y1) were evaluated as sensitive because of the sign of salt injury appeared on the leaves and seedlings become dead (Table 1).

FL478 was proven to be highly tolerant of high-saline environments (Thomson et al. 2010), while IR64 is sensitive to saline conditions (Palao et al. 2013). We obtained similar results under the $150 \mathrm{mM} \mathrm{NaCl}$ stress. These results further confirmed the responses of the rice lines to salt stress. The contrasting responses of the seven rice lines to salt stress caused us to examine their DNA polymorphism at the genome level.

Genome sequencing and sequence read mapping

A total of 7,980,092 high-quality reads were obtained for the seven rice lines, with an average of 1.14 million high-quality reads per line and an average read length of $93 \mathrm{bp}$ (Fig. 1). A quality check showed that $95.71 \%$ of the reads had a quality of $\geq$ Q20. Because both flanking sequences of the BamHI sites were sequenced and it was estimated that there is one BamHI site in approximately $8.5 \mathrm{~kb}$ of the $400-\mathrm{Mb}$ rice genome, the sequence reads for a line had average coverage of 12.1x. The average coverage for SAL-9, SAL-10 SAL-11, SAL-12, FL478, Pokkali and IR64 was 13.0x, 11.5x, 11.5x, 13.0x for, 11.5x, 10.5x, and $13.0 x$ (Table 2). Approximately $83 \%$ of the reads from each line were mapped to the rice Nipponbare reference genome, while the remaining $17 \%$ of the reads were unmapped. The average range of mapped reads was between $66 \%$ for Pokkali and $87 \%$ for SAL-9 (Fig. 1).

Table 2

Statistics of genotyping-by-sequencing results and SNP and InDel variant discovery

\begin{tabular}{|llllllllll|}
\hline Rice line & SAL-9 & SAL-10 & SAL-11 & SAL-12 & FL478 & Pokkali & IR64 & Total & Average \\
\hline Read length (bp) & 95 & 92 & 95 & 93 & 93 & 90 & 94 & 652 & 93 \\
\hline Mapped reads (\%) & 87 & 83 & 85 & 86 & 86 & 66 & 86 & 579 & 83 \\
\hline Read depth (x) & 13.0 & 11.5 & 11.5 & 13.0 & 11.5 & 10.5 & 13.0 & 84 & 12 \\
\hline Raw variants & 32,908 & 27,263 & 36,587 & 32,747 & 36,259 & 22,347 & 37,111 & 225,222 & 32,175 \\
\hline Filtered SNPs & 11,417 & 10,532 & 13,803 & 12,038 & 10,151 & 6,757 & 15,461 & 80,159 & 10,146 \\
\hline Filtered InDels & 857 & 865 & 1,115 & 997 & 778 & 533 & 1,258 & 6,403 & 915 \\
\hline Sequence in Mb & 227.6 & 256.4 & 294.4 & 246.9 & 213.7 & 210.4 & 323.7 & 1,773 & 253.2 \\
\hline
\end{tabular}

Distribution of SNPs and InDels in the rice genome

A total of 225,222 raw variants were identified in the seven rice lines when the rice Nipponbare genome was used as the reference. The raw variants were filtered with a minimum read depth of $10 x$ and mapping quality of $\geq 30$. Consequently, we obtained a total of 80,159 high-quality SNPs and 6,403 high-quality InDels (Table 2). The largest number of variants was found for chromosome $1(9,852)$ and the smallest for chromosome $9(5,328)$. The largest numbers of SNPs and InDels were present in IR64 (15,461 and 1,258) and SAL-11 (13,803 and 1,115), while the smallest numbers were present in Pokkali $(6,757$ and 533) and FL478 (10,151 and 778) (Fig. 2). The average number of variants for each chromosome was 12,366, with one variant for every 30,183 bases (Table S1). Only 186 of the SNPs were common between FL478 and IR64, and 38 were common among FL478, IR64, and Pokkali. IR64/SAL-9 and IR64/SAL-10 had 193 and 143 common SNPs, respectively, while FL478/SAL-9 and FL478/SAL-10 had 213 and 140 common SNPs, respectively. IR64/SAL-11 and IR64/SAL-12 had 228 and 193 common SNPs, respectively; however, FL478/SAL-11 and FL478/SAL-12 had 188 and 223 common SNPs, respectively (Fig. 3).

The average SNP density across the seven rice lines was $3.1 / 100 \mathrm{~kb}$. Chromosome 10 had the highest SNP density, with average $3.7 / 100 \mathrm{~kb}$, while chromosome 4 had the lowest SNP density, with average 2.39/100 kb, and only SAL-11 had the lowest SNP density on chromosome 12 which is $2.84 / 100 \mathrm{~kb}$. The highest average of SNP density was observed in IR64 and SAL-11, which is 4.17/100 kb and 3.71/100 kb, while the lowest average of SNP density was observed in Pokkali and SAL-10, which is $1.82 / 100 \mathrm{~kb}$ and 2.82/100 kb (Table 3). 
Table 3

SNP density among the seven rice lines and their chromosomes

\begin{tabular}{|c|c|c|c|c|c|c|c|c|c|c|c|c|c|}
\hline \multirow[t]{2}{*}{ Chrom. } & \multicolumn{2}{|l|}{ SAL-9 } & \multicolumn{2}{|l|}{ SAL-10 } & \multicolumn{2}{|l|}{ SAL-11 } & \multicolumn{2}{|l|}{ SAL-12 } & \multicolumn{2}{|l|}{ FL478 } & \multicolumn{2}{|c|}{ Pokkali } & \multirow{2}{*}{$\frac{\text { IR }}{\mathrm{SI}}$} \\
\hline & SNPs & $\begin{array}{l}\text { SNPs/100 } \\
\mathrm{kb}\end{array}$ & SNPs & $\begin{array}{l}\text { SNPs/100 } \\
\mathrm{kb}\end{array}$ & SNPs & $\begin{array}{l}\text { SNPs/100 } \\
\mathrm{kb}\end{array}$ & SNPs & $\begin{array}{l}\text { SNPs/100 } \\
\mathrm{kb}\end{array}$ & SNPs & $\begin{array}{l}\text { SNPs/100 } \\
\mathrm{kb}\end{array}$ & SNPs & $\begin{array}{l}\text { SNPs/100 } \\
\mathrm{kb}\end{array}$ & \\
\hline 1 & 1405 & 3.25 & 1261 & 2.91 & 1431 & 3.31 & 1347 & 3.11 & 1140 & 2.63 & 710 & 1.64 & 17 \\
\hline 2 & 1146 & 3.19 & 1134 & 3.16 & 1483 & 4.13 & 1173 & 3.26 & 971 & 2.70 & 709 & 1.97 & 15 \\
\hline 3 & 1208 & 3.32 & 1081 & 2.97 & 1441 & 3.96 & 1289 & 3.54 & 1066 & 2.93 & 679 & 1.86 & $1 \epsilon$ \\
\hline 4 & 789 & 2.22 & 819 & 2.31 & 1073 & 3.02 & 910 & 2.56 & 690 & 1.94 & 473 & 1.33 & 12 \\
\hline 5 & 831 & 2.77 & 751 & 2.51 & 1121 & 3.74 & 905 & 3.02 & 756 & 2.52 & 594 & 1.98 & $1 \mathrm{C}$ \\
\hline 6 & 1088 & 3.48 & 991 & 3.17 & 1305 & 4.18 & 1117 & 3.57 & 968 & 3.10 & 638 & 2.04 & 14 \\
\hline 7 & 876 & 2.95 & 840 & 2.83 & 1126 & 3.79 & 994 & 3.35 & 831 & 2.80 & 572 & 1.93 & 12 \\
\hline 8 & 819 & 2.88 & 747 & 2.63 & 1085 & 3.81 & 863 & 3.03 & 826 & 2.90 & 468 & 1.65 & 12 \\
\hline 9 & 706 & 3.07 & 590 & 2.56 & 809 & 3.52 & 801 & 3.48 & 675 & 2.93 & 420 & 1.83 & 94 \\
\hline 10 & 883 & 3.80 & 818 & 3.52 & 1021 & 4.40 & 895 & 3.86 & 726 & 3.13 & 512 & 2.21 & 12 \\
\hline 11 & 930 & 3.20 & 850 & 2.93 & 1125 & 3.88 & 971 & 3.35 & 847 & 2.92 & 596 & 2.05 & 12 \\
\hline 12 & 736 & 2.67 & 650 & 2.36 & 783 & 2.84 & 773 & 2.81 & 655 & 2.38 & 386 & 1.40 & $1 \mathrm{C}$ \\
\hline $\begin{array}{l}\text { Total/ } \\
\text { average }\end{array}$ & 11,417 & 3.07 & 10,532 & 2.82 & 13,803 & 3.71 & 12,038 & 3.25 & 10,151 & 2.74 & 6,757 & 1.82 & 15 \\
\hline
\end{tabular}

Annotation of variants

Of the SNP and InDel variants, 35,846 were found in genic regions, while the remaining 50,140 variants were found in intergenic regions. Out of the 35,846 variants present in the genic regions, 12,850 were present in introns; 2,228 and 2,106 were present in 5'UTRs and 3'UTRs, respectively; and 18,662 were present in coding sequences (CDSs). The SNP variants in the CDSs were divided into synonymous and non-synonymous. It was found that 10,990 of the SNPs in the CDSs were non-synonymous (nsSNPs) and resulted in changed amino acids. The number of nsSNPs was higher than the synonymous SNPs (7,672) in all seven rice lines analyzed (Table 4). There were 2,101 nsSNPs and InDels common between FL478 and IR64 and 1,372 nsSNPs polymorphic between the two. A total of 566 nsSNPs in the seven rice lines had deleterious effects (SIFT score $<0.05)$ on protein function. The transition (Ts) frequency $(112,686)$ was much higher than that of transversions (Tv) (47,632). The Ts/Tv ratio of the SNPs ranged from 2.2 for SAL-12 to 2.5 for IR64, with an average of 2.0 (Fig. 4).

Table 4

Distribution of SNP and InDel variants in the coding and non-coding regions of the genomes of the seven rice lines

\begin{tabular}{|lllllllll|}
\hline Variant class & SAL-9 & SAL-10 & SAL-11 & SAL-12 & FL478 & Pokkali & IR64 & Total \\
\hline 5'UTR & 340 & 307 & 372 & 328 & 297 & 197 & 387 & 2,228 \\
\hline 3'UTR & 291 & 295 & 365 & 346 & 283 & 175 & 351 & 2,106 \\
\hline Intron & 1,760 & 1,735 & 2,263 & 1,989 & 1,531 & 1,175 & 2,397 & 12,850 \\
\hline Non-synonymous & 1,604 & 1,440 & 1,869 & 1,649 & 1,411 & 992 & 2,025 & 10,990 \\
\hline Synonymous & 1,136 & 1,036 & 1,294 & 1,140 & 980 & 695 & 1,391 & 7,672 \\
\hline Intergenic & 6,987 & 6,444 & 8,551 & 7,388 & 6,267 & 4,547 & 9,956 & 50,140 \\
\hline Total & 12,118 & 11,257 & 14,714 & 12,840 & 10,769 & 7,781 & 16,507 & \\
\hline
\end{tabular}

Effects of nsSNPs on protein functions of abiotic stress-related loci

We identified 116 abiotic stress-related gene loci (between FL478 and IR64) containing a total of 138 nsSNPs in their CDSs, with 1 to 3 nsSNPs per locus, having effects on protein functions (Table S2). Among the 138 nsSNPs, 15 were highly deleterious (SIFT score $=0.0)$ for protein functions.

Genotyping matrices were generated on the nsSNPs. Three nsSNPs were identified in the coding region of LOC_Os02g12820 (helix-loop-helix DNA binding domain-containing protein) on chromosome 2. However, only one of them had a deleterious effect on protein function. This locus had 'A' for the nsSNP at position 6749649 for all three salt stress-sensitive lines (IR64, SAL-11, and SAL-12), while it had 'G' for the nsSNP at this position for all four salt stress-tolerant lines (FL478, Pokkali, SAL-9, and SAL-10), and the same 'G' nucleotide was present in the reference genome (Fig. 5-a). The presence of the T/C nsSNP at position 2927171 between the tolerant lines (FL478, Pokkali, SAL-9, and SAL-10) and sensitive lines (IR64, SAL-11, and SAL-12) in the "flowering locus T" gene (LOC_Os06g06300) was predicted to be deleterious and changed the function of the corresponding amino acid (Fig. 5-b). Further, the T/C nsSNP is present in all three salt stress-sensitive lines, at position 4835996 in LOC_Os10g08940 (Fig. 5-d). Moreover, we also detected a G/A nsSNP in FL478 and Pokkali (the salt stress-tolerant lines) at position 31016149 in the potassium channel protein (LOC_Os03g54100), even though the other five rice lines had no nsSNP at this 
position (Fig. 5-c). These two lines (FL478 and Pokkali) had similar phenotypic expression under salt stress (Table 1). In addition, we observed a G/A nsSNP only in the salt stress-sensitive line IR64, in the "white-brown complex homolog protein 11" gene (LOC_Os12g22284), but no SNP was identified in the other six lines. This gene may be playing a role in transport and response to abiotic stress.

Genetic diversity among the seven rice lines

A phylogenetic tree was constructed using nsSNPs present in the coding regions. Figure 6 shows the genetic relationships among the seven rice lines studied. Two distinct groups were identified that corresponded to the tolerant and sensitive lines, respectively. The tolerant lines SAL-9 and SAL10 were closely related to FL478 and Pokkali, while the sensitive lines SAL-11 and SAL-12 were closely related to the sensitive line IR64 (Fig. 6).

\section{Discussion}

It is necessary to improve and develop rice varieties with a high salinity tolerance to meet the increasing demand for rice. Salinity is one of the significant abiotic stress that affects the rice production. Large tracts of agricultural lands are left unproductive because of the lack of suitable salt-tolerant rice varieties (Pundir et al. 2021). To increase rice production in these barren areas, it is essential to identify novel alleles and genes that control complex quantitative salt tolerance traits in diverse rice germplasm (Kumar et al. 2015). Several studies have identified clearly IR64 to be as sensitive (Sarhadi et al. 2012; Palao et al. 2013) and FL478 as a tolerant variety at the seedling stage (Thomson et al. 2010; Zhao et al. 2014) under high salinity stress conditions. We have sequenced and analyzed in this study the GBS data of five promising salt tolerant rice lines using IR64 and FL478 as the salt stress-sensitive and -tolerant checks, respectively.

A total of 7,980,092-base high-quality reads were obtained for the seven rice lines. Although approximately $83 \%$ of the reads were mapped to the rice Nipponbare reference genome, approximately $17 \%$ were not mapped to the reference genome. This could be largely attributed to the differences between the genomes of japonica and indica subspecies (Tang et al. 2006). Sequencing errors may be another source that caused the unmapped reads, but a high read depth of 12x showed a high quality of sequencing data, as reported earlier (Mehra et al. 2015).

Our study has shown that chromosome 1 has the most significant number of variants because it is the largest in size, while chromosome 9 has the smallest number of variants. This result is consistent with that observed in Swarna, IR64, and Pokkali (Jain et al. 2014; Rathinasabapathi et al. 2015). We have identified 80,159 high-quality SNPs, with an average of 3 SNPs/100 kb, for the seven rice lines. In contrast, an average of 1 SNP/16 kb was observed from GBS in 45 California rice cultivars (Kim and Tai 2013). The difference in SNP density could be due to their sequencing depth and genetic diversity between the genotypes studied, and Nipponbare used as the rice reference genome. The observation of the highest SNP density on chromosome 10 (3.7) and the lowest SNP density on chromosome 4 (2.39) of the seven rice lines resembled that was previously reported in Swarna (Rathinasabapathi et al. 2015). Our study shows that these rice lines have a transitional to transversal SNP ratio varying from 2.2 to 2.5 , which is almost the same as that (from 2.00 to 2.53 ) reported in the rice genome (Hu et al. 2014; Hwang et al. 2014).

A large number of nsSNPs were identified in the CDS regions of the seven rice lines, and it has already been reported in the rice genome that the number of nsSNPs is higher than the number of synonymous SNPs (Mehra et al. 2015; Rathinasabapathi et al. 2015). However, the objective of our study was to target those nsSNPs that affect protein function, especially those associated with responses to salt stress. The amino acid residues that are necessary for the stability of proteins and biological functions are often more conserved than others. Therefore, the presence of nsSNPs in the highly conserved region of a gene might play a key role at the functional level (Arai-Kichise et al. 2011). The SIFT system uses this fact to check the effects of nsSNPs on protein function as deleterious or tolerated (Kumar et al. 2009). Deleterious SNPs affect phenotype by affecting protein synthesis. On the other hand, the SNPs in the non-coding regions could not cause changes in amino acid but might affect the splice and binding sites for transcription factor at the transcriptional level (Xu et al. 2014).

Several candidate genes have been previously identified in rice that might be involved in plant response to salt stress, such as OsNHX1, OsDREB1, SalT, OsHKT1, OsCPK17, and OsLEA5 (Wang et al. 2007; Negrão et al. 2011; Zhou et al. 2016). Our study has identified five new loci whose protein functions are affected by the presence of nsSNPs in their coding regions. We found that $35 \%$ of the nsSNP variants are the same between FL478 and IR64. It might be because both lines are from the same rice subspecies, subsp. indica. One nsSNP, G/A, was identified in the first exon of the HLH gene (LOC_Os02g12820.1). The presence of ' $A$ ' in the salt stress-sensitive lines at position 6749649 of the HLH gene alters protein function by replacing alanine with valine. The functional effect of this nsSNP in the sensitive lines may down-regulate the expression of the protein under salinity stress. The nsSNP identified in the first exon of LOC_Os06g06300 at position 2927171 has resulted in amino acid substitution from valine to alanine. The finding that the gene in Pokkali has 'C' and in IR64 has ' $T$ ' at position 2927171 is the same as previously reported (http://www.nipgr.res.in/mjain.html?page=ricesnp). The presence of the nsSNP in the LOC_Os06g06300 gene in the salt stress-tolerant lines (FL478, Pokkali, SAL-9, and SAL-10) and its absence in the sensitive lines (IR64, SAL-11, and SAL-12) suggest that this gene may be associated with plant response to salt stress and may increase its efficiency under salt stress conditions. The LOC_Os06g06300 gene was previously reported to respond to abiotic stress (Zhang et al. 2016). A genotyping matrix showed allelic variation between the tolerant and sensitive lines at position 4835996 of the LOC_Os10g08940 gene. The presence of ' $C$ ' variant in the salt stress-sensitive lines changed the tTc codon to the tCc codon (Phe to Ser). FL478 and Pokkali that are highly tolerant of high salt stress have an nsSNP in the second exon of the TPKA gene (LOC_Os03g54100) that has changed arginine into tryptophan. Since FL478 was developed from a cross of Pokkali and IR29 (Thomson et al. 2010 ), we conclude that this nsSNP in the TPKA gene is conserved between FL478 and Pokkali. The analysis revealed that the conserved regions for FL478, Pokkali, SAL-9, SAL-10, SAL-11, and SAL-12 are present in the LOC_Os12g22284 locus of chromosome 12, but IR64 has an nsSNP at position 12586623 . IR64 is highly sensitive to salt stress; therefore, the presence of an SNP that has changed the amino acid (Thr to Met) might be down-regulating the function of a protein. Our study reports four loci containing deleterious SNPs in coding regions. Since the deleterious SNPs influence the functions of the gene coding protein, the results provide useful information to carry out further experiments necessary to validate the predicted change in protein function. The presence of these nsSNPs in 
the identified functional genes could be used in forward or reverse genetics for crop improvement. Identifying SNP markers, especially those associated with salt stress tolerance, will be helpful in marker-assisted breeding for the development of superior rice cultivars that are tolerant of salinity stress.

\section{Declarations}

Funding The materials for the study provided under the Green Super Rice Project (ID OPP1130530) funded by the Bill \& Melinda Gates Foundation (BMGF). Higher Education Commission of Pakistan for funding to conduct next-generation sequencing (NGS) of rice genotypes.

Conflicts of interest The authors declare that they have no conflict of interest.

\section{Availability of data and material}

Authors' contributions RT and MA have contributed equally to this work. RT and MA designed the research. JA developed the rice genotypes. RT conducted the salinity experiment. HBZ sequenced the genomic data. RT and MA analyzed the bioinformatic data. RT wrote the manuscript. MA, HBZ and JA revised the manuscript and improved the english writing. All the authors read and approved the final manuscript.

Acknowledgments We are grateful to BMGF for funding the GSR project and the Higher Education Commission of Pakistan for their funding to conduct nextgeneration sequencing (NGS) of rice genotypes.

Consent for publication Not applicable.

\section{References}

1. Agarwal P, Parida SK, Raghuvanshi S, Kapoor S, Khurana P, Khurana JP, Tyagi AK (2016) Rice improvement through genome-based functional analysis and molecular breeding in India. Rice 9:1

2. Al-Tamimi N, Brien C, Oakey H, Berger B, Saade S, Ho YS, Schmöckel SM, Tester M, Negrão S (2016) Salinity tolerance loci revealed in rice using highthroughput non-invasive phenotyping. Nat Commun 7:13342

3. Alexandrov N, Tai S, Wang W, Mansueto L, Palis K, Fuentes RR, Ulat VJ, Chebotarov D, Zhang G, Li Z (2015) SNP-Seek database of SNPs derived from 3000 rice genomes. Nucleic Acids Res 43:D1023-D1027

4. Ali J, Aslam UM, Tariq R, Murugaiyan V, Schnable PS, Li D, Marfori-Nazarea CM, Hernandez JE, Arif M, Xu J (2018) Exploiting the Genomic Diversity of Rice (Oryza sativa L.): SNP-Typing in 11 Early-Backcross Introgression-Breeding Populations. Front Plant Sci 9:849

5. Ali MN, Yeasmin L, Gantait S, Goswami R, Chakraborty S (2014) Screening of rice landraces for salinity tolerance at seedling stage through morphological and molecular markers. Physiol Mol Biol Plants 20:411-423

6. Arai-Kichise Y, Shiwa Y, Nagasaki H, Ebana K, Yoshikawa H, Yano M, Wakasa K (2011) Discovery of genome-wide DNA polymorphisms in a landrace cultivar of japonica rice by whole-genome sequencing. Plant Cell Physiol 52:274-282

7. Batayeva D, Labaco B, Ye C, Li X, Usenbekov B, Rysbekova A, Dyuskalieva G, Vergara G, Reinke R, Leung H (2018) Genome-wide association study of seedling stage salinity tolerance in temperate japonica rice germplasm. BMC Genet 19:2

8. Chung YS, Choi SC, Jun T-H, Kim C (2017) Genotyping-by-sequencing: a promising tool for plant genetics research and breeding. Hortic Environ Biote 58:425-431

9. Cingolani P, Platts A, Wang LL, Coon M, Nguyen T, Wang L, Land SJ, Lu X, Ruden DM (2012) A program for annotating and predicting the effects of single nucleotide polymorphisms, SnpEff: SNPs in the genome of Drosophila melanogaster strain w1118; iso-2; iso-3. Fly 6:80-92

10. DePristo MA, Banks E, Poplin R, Garimella KV, Maguire JR, Hartl C, Philippakis AA, Del Angel G, Rivas MA, Hanna M (2011) A framework for variation discovery and genotyping using next-generation DNA sequencing data. Nat Genet 43:491-498

11. Dereeper A, Homa F, Andres G, Sempere G, Sarah G, Hueber Y, Dufayard J-F, Ruiz M (2015) SNiPlay3: a web-based application for exploration and large scale analyses of genomic variations. Nucleic Acids Res 43:W295-W300

12. Gregorio GB, Senadhira D, Mendoza RD (1997) Screening rice for salinity tolerance. IRRI discussion papers No. 22. International Rice Research Institute, Manila, Philippines

13. Hayashi K, Yoshida H, Ashikawa I (2006) Development of PCR-based allele-specific and InDel marker sets for nine rice blast resistance genes. Theor Appl Genet 113:251-260

14. Hu Y, Mao B, Peng Y, Sun Y, Pan Y, Xia Y, Sheng X, Li Y, Tang L, Yuan L (2014) Deep re-sequencing of a widely used maintainer line of hybrid rice for discovery of DNA polymorphisms and evaluation of genetic diversity. Mol Genet Genomics 289:303-315

15. Hwang S-G, Hwang JG, Kim DS, Jang CS (2014) Genome-wide DNA polymorphism and transcriptome analysis of an early-maturing rice mutant. Genet 142:73-85

16. Jain M, Moharana KC, Shankar R, Kumari R, Garg R (2014) Genomewide discovery of DNA polymorphisms in rice cultivars with contrasting drought and salinity stress response and their functional relevance. Plant Biotechnol J 12:253-264

17. Kawahara Y, de la Bastide M, Hamilton JP, Kanamori H, McCombie WR, Ouyang S, Schwartz DC, Tanaka T, Wu J, Zhou S (2013) Improvement of the Oryza sativa Nipponbare reference genome using next generation sequence and optical map data. Rice 6:4-10

18. Kim S-I, Tai TH (2013) Identification of SNPs in closely related Temperate Japonica rice cultivars using restriction enzyme-phased sequencing. PloS one 8:e60176 
19. Krishnamurthy S, Pundir P, Warraich AS, Rathor S, Lokeshkumar B, Singh NK, Sharma PC (2020) Introgressed saltol QTL lines improves the salinity tolerance in rice at seedling stage. Frontiers in plant science 11:833

20. Kumar P, Henikoff S, Ng PC (2009) Predicting the effects of coding non-synonymous variants on protein function using the SIFT algorithm. Nat Protoc 4:1073-1081

21. Kumar V, Singh A, Mithra SA, Krishnamurthy S, Parida SK, Jain S, Tiwari KK, Kumar P, Rao AR, Sharma S (2015) Genome-wide association mapping of salinity tolerance in rice (Oryza sativa). DNA Res 22:133-145

22. Li H, Durbin R (2009) Fast and accurate short read alignment with Burrows-Wheeler transform. Bioinformatics 25:1754-1760

23. Li H, Handsaker B, Wysoker A, Fennell T, Ruan J, Homer N, Marth G, Abecasis G, Durbin R (2009) The sequence alignment/map format and SAMtools. Bioinformatics 25:2078-2079

24. Mansueto L, Fuentes RR, Borja FN, Detras J, Abriol-Santos JM, Chebotarov D, Sanciangco M, Palis K, Copetti D, Poliakov A (2017) Rice SNP-seek database update: new SNPs, indels, and queries. Nucleic Acids Res 45:D1075-D1081

25. Mehra P, Pandey BK, Giri J (2015) Genome-wide DNA polymorphisms in low Phosphate tolerant and sensitive rice genotypes. Sci Rep 5:13090

26. Mickelbart MV, Hasegawa PM, Bailey-Serres J (2015) Genetic mechanisms of abiotic stress tolerance that translate to crop yield stability. Nat Rev Genet $16: 237-251$

27. Mondal TK, Ganie SA (2014) Identification and characterization of salt responsive miRNA-SSR markers in rice (Oryza sativa). Gene 535:204-209

28. Naveed SA, Zhang F, Zhang J, Zheng T-Q, Meng L-J, Pang Y-L, Xu J-L, Li Z-K (2018) Identification of QTN and candidate genes for Salinity Tolerance at the Germination and Seedling Stages in Rice by Genome-Wide Association Analyses. Sci Rep 8:6505

29. Negrão S, Cecília Almadanim M, Pires IS, Abreu IA, Maroco J, Courtois B, Gregorio GB, McNally KL, Margarida Oliveira M (2013) New allelic variants found in key rice salt-tolerance genes: an association study. Plant Biotechnol J 11:87-100

30. Negrão S, Courtois B, Ahmadi N, Abreu I, Saibo N, Oliveira M (2011) Recent updates on salinity stress in rice: from physiological to molecular responses. Crit Rev Plant Sci 30:329-377

31. Palao CDC, Viña CBD, Gregorio GB, Singh RK (2013) A new phenotyping technique for salinity tolerance at the reproductive stage in rice. Oryza 50:199207

32. Peterson BK, Weber JN, Kay EH, Fisher HS, Hoekstra HE (2012) Double digest RADseq: an inexpensive method for de novo SNP discovery and genotyping in model and non-model species. PloS one 7:e37135

33. Pundir P, Devi A, Krishnamurthy S, Sharma PC, Vinaykumar N (2021) QTLs in salt rice variety CSR10 reveals salinity tolerance at reproductive stage. Acta Physiol Plant 43:1-15

34. Rathinasabapathi P, Purushothaman N, Ramprasad V, Parani M (2015) Whole genome sequencing and analysis of Swarna, a widely cultivated indica rice variety with low glycemic index. Sci Rep 5:11303

35. Robinson JT, Thorvaldsdóttir H, Winckler W, Guttman M, Lander ES, Getz G, Mesirov JP (2011) Integrative genomics viewer. Nat Biotechnol 29:24-26

36. Sahu PK, Sao R, Mondal S, Vishwakarma G, Gupta SK, Vinay K, Sudhir S, Deepak S, K DB (2020) Next generation sequencing based forward genetic approaches for identification and mapping of causal mutations in crop plants: A comprehensive review. Plants 9:1355

37. Sarhadi E, Bazargani MM, Sajise AG, Abdolahi S, Vispo NA, Arceta M, Nejad GM, Singh RK, Salekdeh GH (2012) Proteomic analysis of rice anthers under salt stress. Plant Physiol Biochem 58:280-287

38. Tang T, Lu J, Huang J, He J, McCouch SR, Shen Y, Kai Z, Purugganan MD, Shi S, Wu C-I (2006) Genomic variation in rice: genesis of highly polymorphic linkage blocks during domestication. PLoS Genet 2:e199

39. Tariq R, Ali J, Arif M (2019) Morphological Screening and SalTo/ Region Based SSR Markers Analysis of Rice (Oryza sativa) Genotypes for Salinity Tolerance at Seedling Stage. Int J Agr Biol 21:25-33

40. Thomson MJ, de Ocampo M, Egdane J, Rahman MA, Sajise AG, Adorada DL, Tumimbang-Raiz E, Blumwald E, Seraj ZI, Singh RK (2010) Characterizing the Saltol quantitative trait locus for salinity tolerance in rice. Rice 3:148-160

41. Tiwari S, Krishnamurthy S, Kumar V, Singh B, Rao A, Rai V, Singh AK, Singh NK (2016) Mapping QTLs for Salt Tolerance in Rice (Oryza sativa L.) by Bulked Segregant Analysis of Recombinant Inbred Lines Using 50K SNP Chip. PloS one 11:e0153610

42. Wang H, Wang H, Shao H, Tang X (2016) Recent Advances in Utilizing Transcription Factors to Improve Plant Abiotic Stress Tolerance by Transgenic Technology. Front Plant Sci 7

43. Wang X-S, Zhu H-B, Jin G-L, Liu H-L, Wu W-R, Zhu J (2007) Genome-scale identification and analysis of LEA genes in rice (Oryza sativa L.). Plant Sci $172: 414-420$

44. Xu J, Yuan Y, Xu Y, Zhang G, Guo X, Wu F, Wang Q, Rong T, Pan G, Cao M (2014) Identification of candidate genes for drought tolerance by whole-genome resequencing in maize. BMC Plant Biol 14:1

45. Zhang C, Liu J, Zhao T, Gomez A, Li C, Yu C, Li H, Lin J, Yang Y, Liu B, Lin C (2016) A Drought-Inducible Transcription Factor Delays Reproductive Timing in Rice. Plant Physiol 171:334-343

46. Zhao K, Tung C-W, Eizenga GC, Wright MH, Ali ML, Price AH, Norton GJ, Islam MR, Reynolds A, Mezey J (2011) Genome-wide association mapping reveals a rich genetic architecture of complex traits in Oryza sativa. Nat Commun 2:467

47. Zhao X, Wang W, Zhang F, Deng J, Li Z, Fu B (2014) Comparative metabolite profiling of two rice genotypes with contrasting salt stress tolerance at the seedling stage. PloS one 9:e108020

48. Zhao Y, Wang K, Wang W-I, Yin T-t, Dong W-q, Xu C-j (2019) A high-throughput SNP discovery strategy for RNA-seq data. BMC Genom 20:160 
49. Zhou Y, Yang P, Cui F, Zhang F, Luo X, Xie J (2016) Transcriptome Analysis of Salt Stress Responsiveness in the Seedlings of Dongxiang Wild Rice (Oryza rufipogon Griff.). PloS one 11:e0146242

Figures

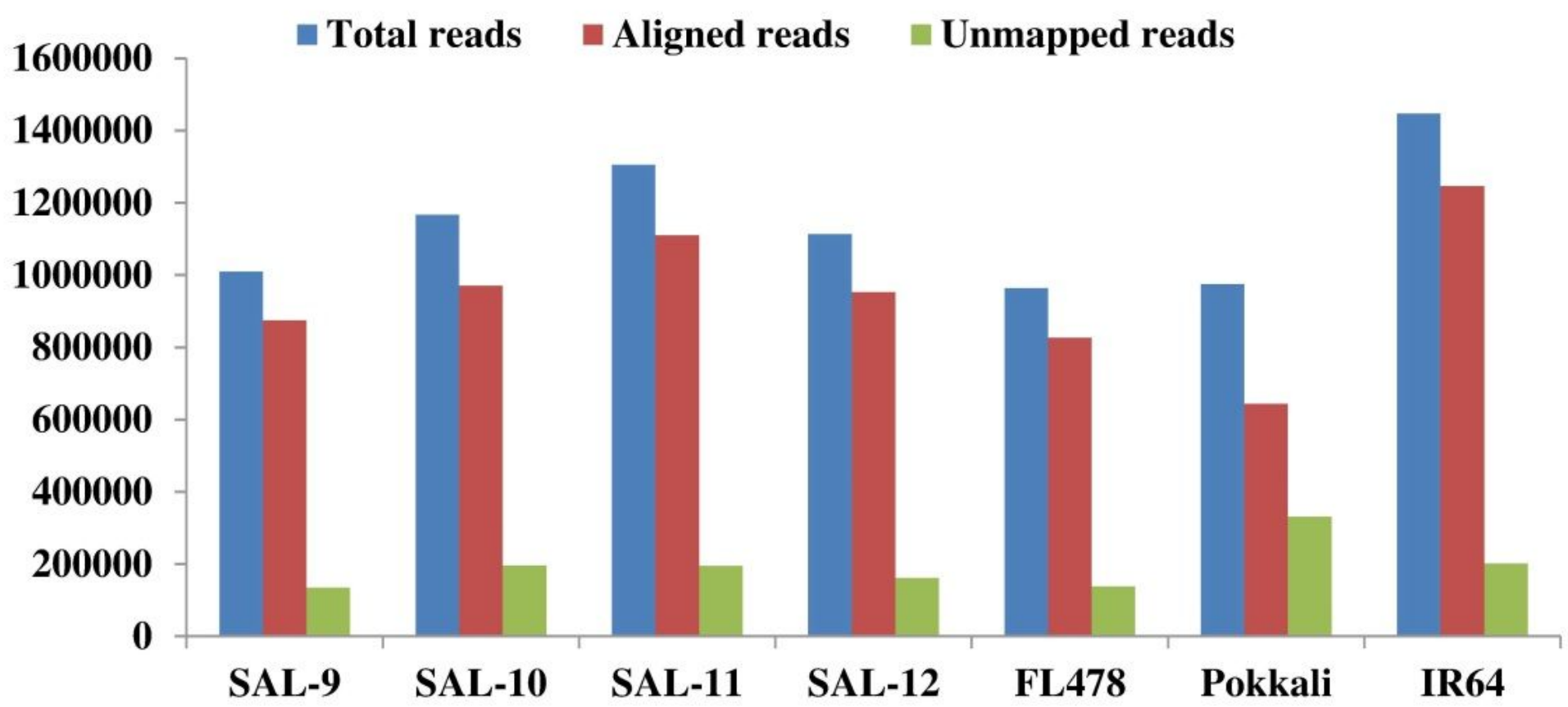

Figure 1

Sequence reads and their mapping to the rice reference genome 

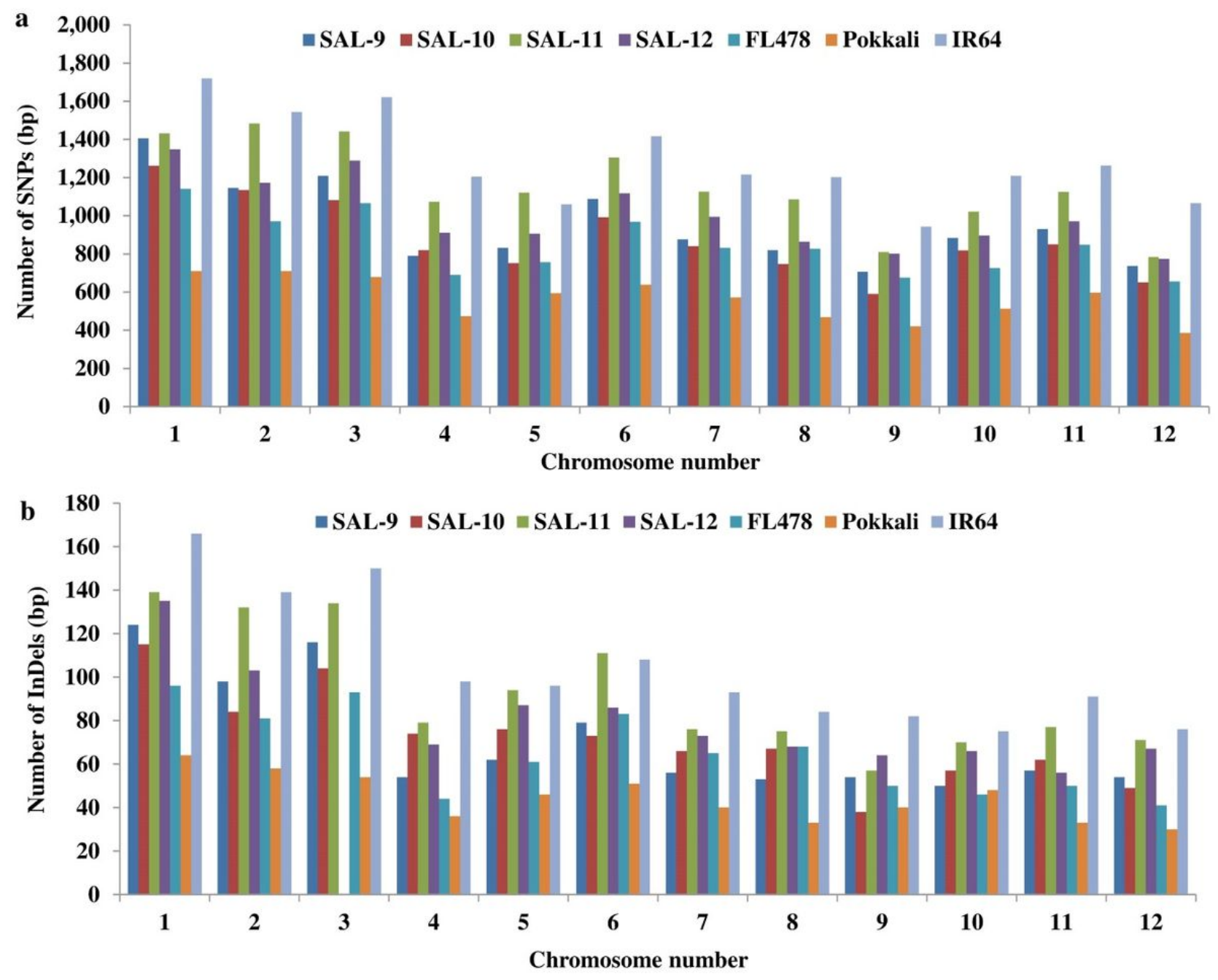

Figure 2

Distribution of SNPs (a) and InDels (b) on the rice chromosomes.

The different color bars show the numbers of SNPs and InDels in the seven rice lines 


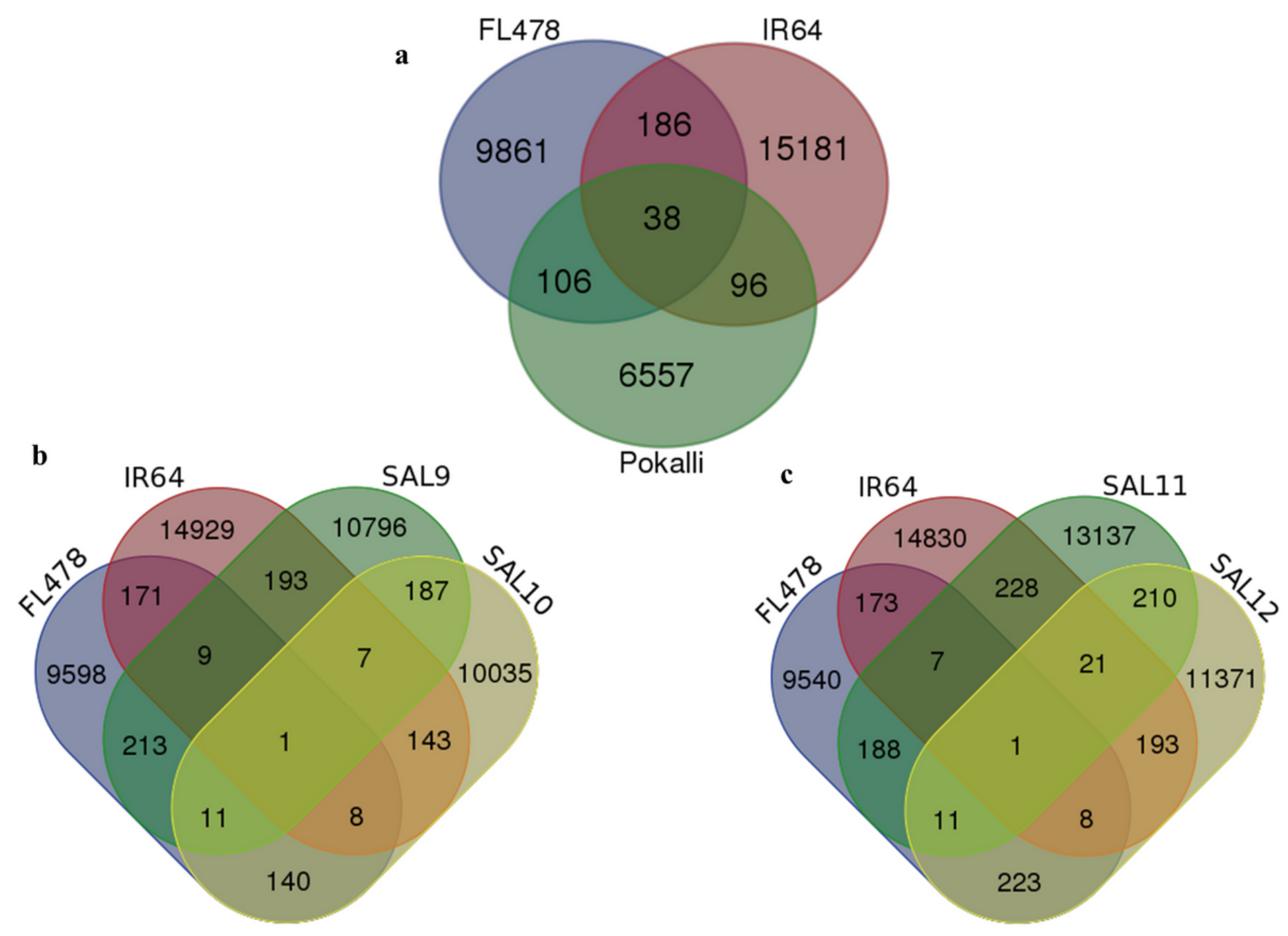

Figure 3

Venn diagrams of specific and common SNPs in seven rice genotypes 


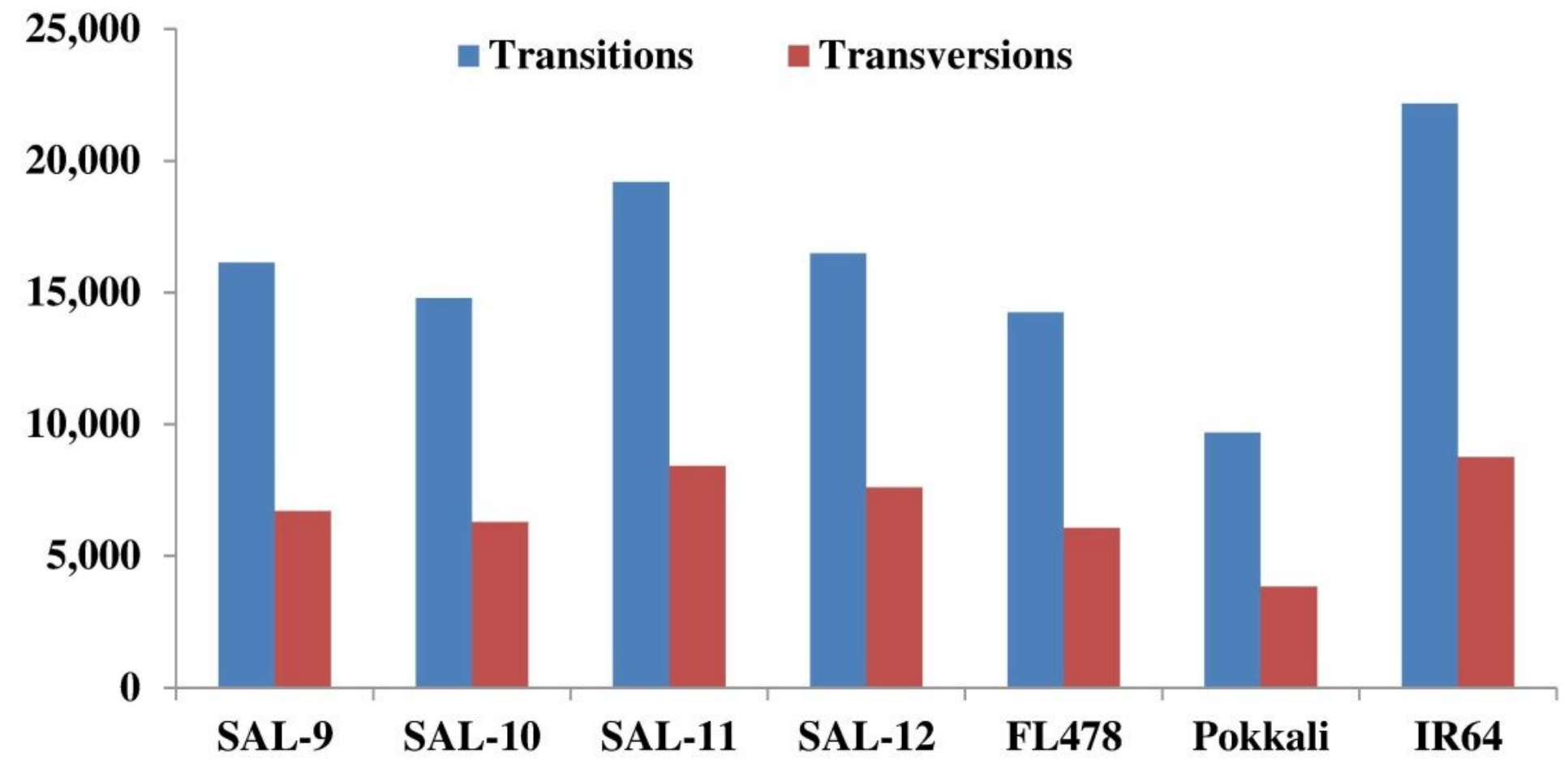

Figure 4

Frequencies of transitional and transversal SNPs in the genomes of the seven rice lines 

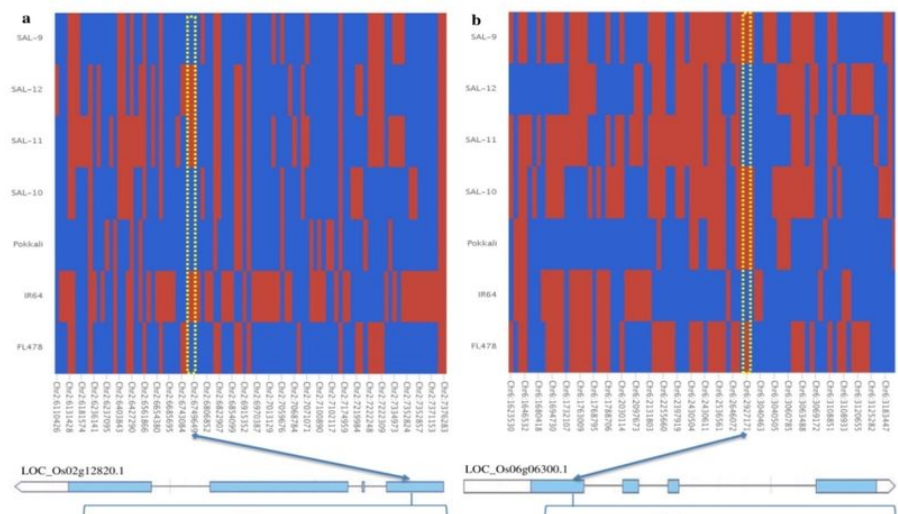

SAL-9 CGCGTCGAACAGGGGGCGCACGCAGCCGCC $\begin{array}{ll}\text { SAL-12 } & \text { CGCGTCGAACAGGAEGCGCACGCAGCCGCC } \\ \text { SAL-11 CGCGTCGAACAGCAEGCGCACGCAGCCGCC }\end{array}$

SAL-11 CGCGTCGAACAGY ACGCGCACGCAGCCGCC

Pokkali CGCGTCGAACAGd GEGCGCACGCAGCCGCC

$\begin{array}{ll}\text { IR64 } & \text { CGCGTCGAACAG A GCGCACGCAGCCGCC } \\ \text { FL478 } & \text { CGCGTCGAACAGCGEGCGCACGCAGCCGCC } \\ \text { Reference CGCGTCGAACAGdGEGCGCACGCAGCCGCC }\end{array}$

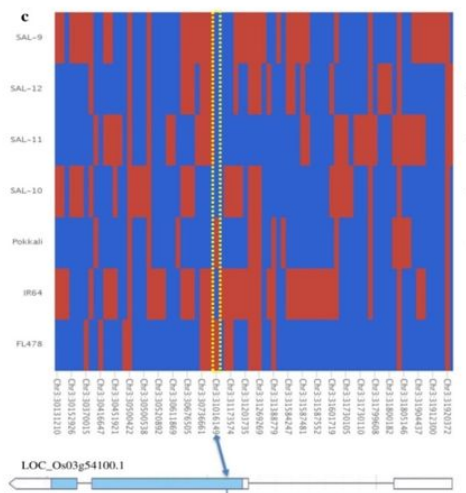

SAL-9 AaCCTCAGTCCEAGCTATGGTGCAAGGATC SAL-12 AACCTCAGTCTCAGCTATGGTGCAAGGATC SAL-1 AACCTCAGTG Pokkali AACCTCAGTOCAGCTATGGTGCAAGGATC IR64 AACCTCAGTCTEAGCTATGGTGCAAGGATC FL478 AACCTCAGTCCEAGCTATGGTGCAAGGATC

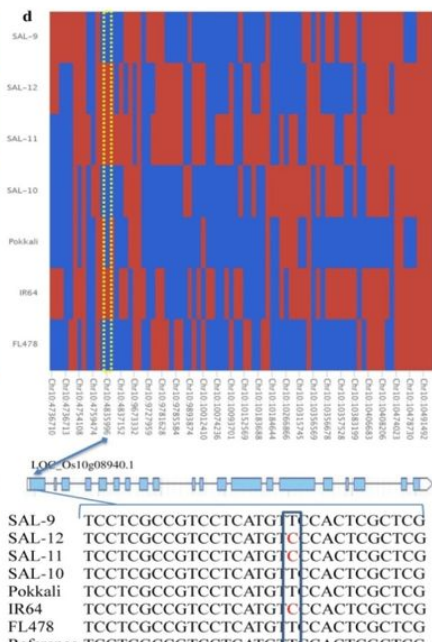

SAL-9 CTGACGGCTTCCG TGCAGCACATTAGGGT

SAL-11 CTGACGGCTTCOG TCAGCACATTAGGG

SAL-10 CTGACGGCTTCOG TGCAGCACATTAGGG

Pokkali CTGACGGCTTC ACTGCAGCACATTAGGG

FL478 CTGACGGCTTCO GTGCAGCACATTAGGG

Reference CTGACGGCTTC G TGCAGCACATTAGGGT

\section{Figure 5}

Genotyping matrices of the seven rice lines for the genes containing protein function-influenced nsSNPs, LOC_Os02g12820 (a), LOC_Os06g06300 (b), LOC_Os03g5400 (c), and LOC_Os10g08940 (d).

Red regions show the nsSNP variants and blue regions show the identical bases with respect to the reference genome. Vertical yellow dashed lines represent the position of the nsSNPs. Arrows indicate the locations of nsSNPs within the CDS that discriminate tolerant genotypes from sensitive ones 
-Pokalli

-FL478

-SAL-9

-SAL-10

-SAL-12

-SAL-11

$\cdot$-IR64

0 0.1

Figure 6

Phylogenetic tree showing the genetic relationships among the seven rice lines constructed based on nsSNPs.

The sensitive lines are represented by red color and the tolerant lines by green color

\section{Supplementary Files}

This is a list of supplementary files associated with this preprint. Click to download.

- SupplementalMaterial.doc 